Annuaire du Collège de France 2017-2018

\title{
Épigénétique et mémoire cellulaire
}

\section{Edith Heard}

\section{(2) OpenEdition}

Journals

Édition électronique

URL : https://journals.openedition.org/annuaire-cdf/15564

DOI : $10.4000 /$ annuaire-cdf. 15564

ISBN : 978-2-7226-0572-5

ISSN : 2109-9227

Éditeur

Collège de France

Édition imprimée

Date de publication : 30 décembre 2020

Pagination : 125-129

ISBN : 978-2-7226-0516-9

ISSN : 0069-5580

Référence électronique

Edith Heard, «Épigénétique et mémoire cellulaire », L'annuaire du Collège de France [En ligne], 118 | 2020, mis en ligne le 01 avril 2021, consulté le 22 août 2022. URL : http://journals.openedition.org/ annuaire-cdf/15564 ; DOI : https://doi.org/10.4000/annuaire-cdf.15564 


\title{
ÉPIGÉNÉTIQUE ET MÉMOIRE CELLULAIRE
}

\author{
Edith HEARD \\ Professeure au Collège de France
}

Mots-clés : chromosome $X$, génétique, épigénétique

La série de cours "Le chromosome $X$, paradigme de la génétique et de l'épigénétique » est disponible, en audio et en vidéo, sur le site internet du Collège de France (https://www.college-de-france.fr/site/edith-heard/course-2017-2018.htm).

\section{ENSEIGNEMENT}

COURS - LE CHROMOSOME X, PARADIGME DE LA GÉNÉTIQUE ET DE L'ÉPIGÉNÉTIQUE

\section{Cours 1 - Chromosomes sexuels et compensation de dose}

Dans ce premier cours, je présente les divers mécanismes, génétiques et épigénétiques, qui ont été exploités au cours de l'évolution pour déterminer le sexe dans différents organismes. Chez les mammifères, où un système $\mathrm{XX}$ (femelle)/XY (mâle) existe, le facteur de transcription Sry détermine le sexe masculin alors que l'absence de Sry et le récepteur d'hormone nucléaire DAX1 contribue au sexe féminin. Néanmoins, les règles semblent complexes car les recherches récentes indiquent qu'un équilibre délicat existe entre les voies de signalisation masculines et féminines pour la détermination du sexe. Par ailleurs, cet équilibre semble être différent au cours de l'embryogenèse et à l'âge adulte. Ensuite, j'analyse la façon dont les différences de contenu génétique entre les mâles et femelles peuvent poser un problème de dosage. Le chromosome $\mathrm{Y}$ porte souvent beaucoup moins de gènes que le chromosome $\mathrm{X}$ - la présence de deux chromosomes $\mathrm{X}$ chez les femelles versus un chez les mâles peut avoir des effets néfastes. Nous savons que les monosomies ou trisomies chromosomiques sont presque toujours mortelles pour les organismes normaux. Par ailleurs, les modifications du nombre de copies d'un seul gène peuvent causer des problèmes (insuffisance haploïde). Par conséquent, le maintien d'un dosage correct des gènes à plusieurs échelles (des gènes uniques aux chromosomes 
entiers) est important. Des stratégies diverses de compensation de dose ont évolué chez différentes espèces. L'inactivation d'un des deux chromosomes $X$ chez les individus XX (avec ou sans Y) est la stratégie exploitée chez les mammifères.

\section{Cours 2 et 3 - Régulation génétique et épigénétique du chromosome $X$ inactif et dynamique de l'hétérochromatine facultative}

Dans ces deux cours, je présente la régulation épigénétique du chromosome X. Je commence le cours en présentant les bases moléculaires des stratégies de compensation de dose dans différentes espèces XX/XY, comme la mouche de vinaigre, Drosophila melanogaster et le nématode Caenorabditis elegans, afin de comparer ces stratégies de compensation de dose très différentes, avec l'inactivation $\mathrm{du} \mathrm{X}$ chez les mammifères. La détermination du sexe et la compensation de dose sont déclenchées par la même voie chez la drosophile et $C$. elegans - mais pas chez les mammifères où la dose XX déclenche l'inactivation d'un $X$, alors que la présence du gène Sry déclenche le sexe masculin. En 1961, Mary Lyon a proposé, sur la base d'études génétiques chez la souris, que chez les mammifères femelles, l'un des deux chromosomes X devient inactif, et forme le corpuscule de Barr. Plus précisément, elle a émis l'hypothèse qu'au début du développement embryonnaire, soit le chromosome $\mathrm{X}$ paternel, soit le chromosome $\mathrm{X}$ maternel est inactivé dans chaque cellule et que cet état inactif est alors propagé à travers les divisions cellulaires conduisant à la mosaïque cellulaire chez les femelles pour l'expression des gènes liés à l'X. L'hypothèse de Lyon dans son article de 1961marquait le début du domaine de l'inactivation du X ainsi qu'une renaissance dans le domaine de l'épigénétique. Ce processus, à l'intersection de la biologie du développement et de la génétique, est rapidement devenu un paradigme pour l'épigénétique ainsi que pour la formation de l'hétérochromatine. Grace aux études sur le chromosome X inactif, on pourrait comprendre comment l'organisation de la chromatine influence la fonction du génome et l'expression des gènes dans un contexte développemental. Dans ce cours, je présente l'état de nos connaissances actuelles sur ce processus épigénétique á l'échelle d'un chromosome entier. En particulier, je souligne le fait que certains gènes peuvent échapper à l'inactivation suggérant que certains gènes sont plus sensibles à la dose que d'autres - et cela peut varier d'un individu à l'autre et dans différents tissus. Les différences de dose, et ces variations d'expression des gènes sur le $\mathrm{X}$, pourraient avoir des conséquences importantes pour les différences entre les hommes et les femmes - et entre les femmes. Les cours suivants se focalisent sur ces considérations.

\section{Cours 4 - Les troubles neurologiques liés au chromosome $X$}

De nombreux gènes liés au chromosome $X$ chez les mammifères sont impliqués dans les déficiences intellectuelles (XLID). Dans ce cours, j'étudie certains de ces gènes, comme Mecp2, dont l'absence est très nocive (létalité chez les mâles ; syndrome de Rett chez les femelles), mais la double dose est aussi délétère chez les garçons avec une duplication Mecp2. Ceci illustre à quel point le dosage précis est important.

J'analyse également les avantages sélectifs liés aux différences de dose des gènes liés au chromosome $\mathrm{X}$ pour les fonctions spécifiques du cerveau chez les hommes et les femmes. Bien que les hormones aient dominé les théories des origines des différences sexuelles, au cours des deux dernières décennies, le déséquilibre sexuel des effets des chromosomes $\mathrm{X}$ et $\mathrm{Y}$ a été clairement démontré comme causant des 
différences sexuelles dans les tissus non gonadiques qui ne sont pas médiées par les hormones gonadiques. Des gènes spécifiques liés au chromosome $\mathrm{X}$ sont des candidats de choix pour ces effets.

Je décris comment l'XCI aléatoire ainsi que l'échappement variable de certains gènes résultent en une mosaïque cellulaire au sein des individus $\mathrm{XX}$, et entre eux. Chaque femelle est une mosaïque unique pour l'expression génique liée au chromosome $\mathrm{X}$ : le résultat dépendra de l'interaction de tous les allèles entre les deux X (actifs), ainsi que de la variation inhérente de l'initiation de l'inactivation «aléatoire », également dans le mélange et la migration cellulaire. Dans ce cours, j'étudie les avantages du XCI aléatoire par rapport à l'hétérozygotie simple dans le cerveau et la façon dont les possibilités fonctionnelles augmentent en combinant des phénotypes cellulaires. J'analyse également certaines situations dans lesquelles, à l'inverse, il y a une incompatibilité entre cellules exprimant l'un ou l'autre allèle d'un gène lié au chromosome X (le récepteur de l'éphrine et son ligand EFNB1). Ceci peut conduire à certains troubles cranio-faciaux ainsi qu'à une forme d'épilepsie spécifique aux femmes.

Les gènes qui échappent à l'inactivation du $\mathrm{X}$ sont particulièrement présents dans le contexte de maladies spécifiques aux femmes. Certains gènes y échappent toujours, alors que d'autres varient d'un individu à l'autre et à l'intérieur d'un même individu. Les gènes qui y échappent de manière constitutive sont conservés (par exemple : Jarid1c, Utx). Les gènes qui y échappent de manière variable sont moins conservés. Le moment et le lieu de l'échappement semblent aussi importants : la posologie peut varier au cours du développement et chez les adultes. Plusieurs de ces gènes sont impliqués dans des maladies auto-immunes.

\section{Cours 5 - Le chromosome $\mathrm{X}$ et les maladies auto-immunes}

Dans ce dernier cours, j'étudie le fait que la plupart des maladies humaines présentent des différences de sexe spécifiques en matière de prévalence, d'âge d'apparition et/ou de gravité. Ceci inclut les troubles neurologiques et psychiatriques ; les maladies cardiovasculaires qui sont prédominantes chez les hommes à l'âge adulte, mais plus fréquentes chez les femmes ménopausées que chez les hommes ; l'asthme, qui est plus répandu chez les garçons dans l'enfance, mais qui est plus fréquent chez les filles à la puberté et après; plusieurs types de cancer. Enfin, les maladies auto-immunes constituent le cas le plus frappant car elles sont beaucoup plus répandues chez les femmes tout au long de leur vie, mais plus particulièrement dans le cas des maladies qui débutent pendant ou immédiatement après les années de reproduction. Il s'avère que le chromosome $\mathrm{X}$ possède la plus forte densité de gènes liés au système immunitaire parmi tous les chromosomes. Il semble important que ces gènes soient régulés correctement, en particulier dans les cellules du système immunitaire. Dans la maladie du lupus, les niveaux d'expression plus élevés (comme les récepteurs Toll-like 7, Tlr7) ont été observés pour certains de ces gènes. Ce changement d'expression modifie le comportement des cellules et, dans le cas du lupus, ce changement est associé à des phénotypes de maladies auto-immunes beaucoup plus graves. Les études récentes dans les cellules du système immunitaire démontre une dynamique remarquable de l'expression des gènes liés à l'X chez les femelles, due à une plasticité épigénétique inattendue dans certaines lignages adultes. Les recherches sont en cours pour mieux comprendre les mécanismes moléculaires qui conduisent à une telle plasticité. Ceci ouvre une nouvelle ère de 
recherche dans le domaine de la compensation de dose pour les chromosomes sexuels et l'étude de la variation génétique et épigénétique, qui sont responsables des différences entre individus.

\section{Colloque - Le CHROMOSOME X, PARADIGME de LA GÉNÉTIQUE ET DE L'ÉPIGÉNÉTIQUE}

Colloque organisé au Collège de France le 14 mai 2018 par Edith Heard et Agnese Loda :

- Philip Avner (EMBL Rome, Italie)

- Christine Disteche (University of Washington, Seattle, États-Unis)

- Edda Schulz (Max Planck Institute, Berlin, Allemagne)

- James Turner (Crick Institute, Londres)

- Gabriel Marais (CNRS/université Lyon 1)

- Montserrat Anguera (University of Pennsylvania School, États-Unis)

- Claire Rougeulle (unité d'épigénétique et destin cellulaire, Paris, France)

- Joost Gribnau (Erasmus, Rotterdam, Pays-Bas)

\section{RECHERCHE}

Depuis 2010, je dirige l'unité de Génétique et biologie du développement à l'Institut Curie (Paris) composé de neuf équipes, dont la mienne. L'ambition de cette unité repose sur un concept simple, mais fondamental : mieux connaître les processus qui régissent le développement normal pour identifier l'origine des désordres pathologiques. Cette unité fournit une trame multithématique et multidisciplinaire unique pour l'étude des événements qui affectent l'identité cellulaire dans un contexte développemental. J'anime une équipe de recherche au sein de cette unité. Notre objectif est de comprendre comment, au cours du développement et de la différenciation cellulaire, l'acquisition de caractéristiques cellulaires spécialisées est assurée non pas par un changement de la nature et de la séquence des gènes, mais par la manière dont ces gènes sont exprimés. Le développement embryonnaire précoce des mammifères femelles s'accompagne de l'inactivation transcriptionnelle de l'un de leurs deux chromosomes $\mathrm{X}$, achevant ainsi la compensation de dose vis-à-vis des mâles XY. Ce processus, connu sous le nom d'inactivation du chromosome X, représente un paradigme de l'épigenèse développementale. En étudiant le contrôle de l'inactivation du chromosome $\mathrm{X}$, nous développons des méthodes et des techniques permettant la compréhension de mécanismes fondamentaux qui soustendent la régulation de l'expression des gènes, à la fois au cours du développement et de la différenciation cellulaire mais aussi lors de la tumorigenèse. L'inactivation $\mathrm{du}$ chromosome $\mathrm{X}$ est un modèle de choix pour décrypter les mécanismes moléculaires mis en jeu lors de la prise de décisions développementales, ainsi que pour assurer leur maintien. Notre recherche est organisée autour de quatre axes principaux de recherche :

1) Quels sont les mécanismes contrôlant l'initiation de l'inactivation du chromosome X?

2) Comment la répression transcriptionnelle du chromosome $X$ est-elle établie ? 
3) Comment l'état inactif est-il fidèlement transmis au cours des générations cellulaires?

4) Comment le développement tumoral affecte-t-il le maintien de l'état inactif du chromosome $\mathrm{X}$ ?

\section{PuBlications}

Bemmel J.G. van, Galupa R., Gard C., Servant N., Picard C., Davies J., SZempruch A.J., Zhan Y., Żylicz J.J., Nora E.P., Lameiras S., Wit E. de, Gentien D., Baulande S., Giorgetti L., Guttman M., Hughes J.R., Higgs D.R., Gribnau J. et Heard E., « The bipartite TAD organization of the X-inactivation center ensures opposing developmental regulation of Tsix and Xist », Nature Genetics, vol. 51, n ${ }^{\circ}$ 6, 2019, p. 1024-1034, DOI : 10.1038/s41588-019-0412-0.

Borensztein M., Syx L., Servant N. et Heard E., «Transcriptome profiling of single mouse oocytes ", Mouse Oocyte Development, vol. 1818, 2018, Methods in Molecular Biology, p. 51-65, DOI : 10.1007/978-1-4939-8603-3_7.

Barros de Andrade E Sousa L., Jonkers I., Syx L., Dunkel I., Chaumeil J., PiCARD C., Foret B., Chen C.-J., T Lis J., Heard E., Schulz E. et Marsico A., « Kinetics of Xistinduced gene silencing can be predicted from combinations of epigenetic and genomic features », Genome research, vol. 29, 2019, DOI : 10.1101/gr.245027.118.

GALUPA R. et HEARD E., «X-Chromosome inactivation: A crossroads between chromosome architecture and gene regulation », Annual Review of Genetics, vol. 52, n ${ }^{\circ}$ 1, 2018, p. 535-566, DOI : 10.1146/annurev-genet-120116-024611.

Gdula M.R., Nesterova T.B., Pintacuda G., Godwin J., Zhan Y., Ozadam H., McClellan M., Moralli D., Krueger F., Green C.M., Reik W., Kriaucionis S., HEARD E., DEKKER J. et BROCKDORFF N., "The non-canonical SMC protein SmcHD1 antagonises TAD formation and compartmentalisation on the inactive X chromosome ", Nature Communications, vol. 10, $\mathrm{n}^{\circ}$ 1, 2019, p. 30, DOI : 10.1038/s41467-018-07907-2.

Masui O., Heard E. et KoseKi H., «Live imaging of Xist RNA », in T. SAdo (dir.), $X$-Chromosome Inactivation : Methods and Protocols, New York, Springer, coll. « Methods in Molecular Biology », 2018, p. 67-72, DOI : 10.1007/978-1-4939-8766-5_6.

Mutzel V., OKamoto I., Dunkel I., Saitou M., Giorgetti L., Heard E. et Schulz E.G., «A symmetric toggle switch explains the onset of random X inactivation in different mammals ", Nature Structural \& Molecular Biology, vol. 26, $\mathrm{n}^{\circ}$ 5, 2019, p. 350-360, DOI : 10.1038/s41594-019-0214-1.

POLLEX T. et HEARD E., « Nuclear positioning and pairing of X-chromosome inactivation centers are not primary determinants during initiation of random X-inactivation », Nature Genetics, vol. 51, nº 2, 2019, p. 285-295, DOI : 10.1038/s41588-018-0305-7.

PROChIANTZ A. et HEARD E., «France, a refuge for researchers in danger», Medecine Sciences, vol. 34, no 8-9, 2018, p. 635-636, DOI : 10.1051/medsci/20183408001.

Servant N., VAroquauX N., Heard E., Barillot E. et Vert J.-P., « Effective normalization for copy number variation in Hi-C data », BMC Bioinformatics, vol. 19, $\mathrm{n}^{\circ}$ 1, 2018, p. 313, DOI : 10.1186/s12859-018-2256-5.

ŻYlicz J.J., Bousard A., Žumer K., DOSSIN F., MOHAMMad E., DA ROCHA S.T., Schwalb B., Syx L., Dingli F., Loew D., Cramer P. et Heard E., "The implication of early chromatin changes in X chromosome inactivation ", Cell, vol. 176, $\mathrm{n}^{\mathrm{0}} 1-2,2019$, p. 182-197.e23, DOI : 10.1016/j.cell.2018.11.041. 
